\title{
Enhanced Optical and Electrical Properties of ITO/Ag/AZO Transparent Conductors for Photoelectric Applications
}

\author{
Gyeong-Nam Lee, Ponnamma Machaiah M., Wang-Hee Park, and Joondong Kim \\ Department of Electrical Engineering, Incheon National University, Incheon 406772, Republic of Korea \\ Correspondence should be addressed to Joondong Kim; joonkim@incheon.ac.kr
}

Received 9 April 2017; Revised 2 August 2017; Accepted 24 August 2017; Published 22 October 2017

Academic Editor: Jegadesan Subbiah

Copyright (C) 2017 Gyeong-Nam Lee et al. This is an open access article distributed under the Creative Commons Attribution License, which permits unrestricted use, distribution, and reproduction in any medium, provided the original work is properly cited.

\begin{abstract}
The enhancement of the optical and electrical properties of TCO films was investigated by depositing different layers of AZO $(100 \mathrm{~nm}), \mathrm{Ag}(5 \mathrm{~nm}) / \mathrm{AZO}(95 \mathrm{~nm})$, and ITO $(45 \mathrm{~nm}) / \mathrm{Ag}(5 \mathrm{~nm}) / \mathrm{AZO}(50 \mathrm{~nm})$ upon $\mathrm{n}-\mathrm{Si}$ substrate at room temperature by magnetron sputtering method. The ITO/Ag/AZO device efficiently improved the electrical and optical properties with the low sheet resistance of $2.847 \Omega /$ sq. and an increase in the rectification ratio of $455.60 \%$ when compared with AZO and Ag/AZO devices. The combination of ITO/Ag/AZO provided the optimum results in all the electrical and optical properties. These results showed that within the optimized thickness range of $100 \mathrm{~nm}$, compared to AZO and Ag/AZO, ITO/Ag/AZO device showed the improvement for both optical and electrical properties at room temperature.
\end{abstract}

\section{Introduction}

The rapid growth of photovoltaic (PV) devices dominates the industry today due to its meritorious effect on the environment by harvesting solar energy from the sun. This includes superior efficiency, competitive cost to produce electricity, and long-term reliability $[1,2]$. Recently, significant work has been put forward to produce low-cost and highly efficient photoelectric devices using thin film technology imbibing the heterojunction concept.

The heterojunction devices are the devices in which the interface is between two solid-state materials having different structural and optical properties. One such use of the heterojunction devices is the implementation of transparent conducting oxide (TCO) layers in the Si-based devices, and it is one of the important developments in the ongoing progress of photovoltaic research [3].

TCO materials have attracted much attention for its significance in optical and electrical applications from the displays to the thin film solar cell technology and are used as transparent conductive electrodes in virtually every thin film solar cell design and single crystal cells. These coatings can reduce energy loss by as much as $30 \%$ [4]. Thus, these qualities make TCO a promising material which can improve the efficiency of the photoelectric devices. Among all the TCOs, indium tin oxide (ITO) and aluminum zinc oxide (AZO) are widely used in commercial applications such as displays, solar cells, and photodiodes. ITO is known for its excellent electrical conductivity and AZO for its improved optical transparency and thermal stability [5-8]. Hence, to achieve both properties of enhanced transparency and good conductivity in a single device, various combinations of layered TCOs were developed. Kim et al. reported that the bilayered ITO/AZO exhibited higher optical transparency and electrical conductivity than those of single-layer ITO and AZO films.

Hence, it was understood that overall functionalities of bilayer TCO structures were better than single-layer TCOs. However, TCO layers offer relatively high resistivity and low transmittance at room temperature, and the charge carrier generation and separation process still lags behind which lead to the reduction of photoresponse and rectification properties in the device [9]. In this aspect, insertion of a thin metal layer such as $\mathrm{Ag}, \mathrm{Au}$, and $\mathrm{Ni}$ in combination with TCOs has shown to be an effective candidate for the reduction of sheet resistivity. When the light is incident on these 
inserted metals in between the TCO materials, the free electrons available in the metal layer speed up the light transportation from the top to the bottom of the device. Also, the metal layer helps to thrust up the separated charge carriers swiftly to the top electrode [10].

Several combinations of TCO/metal/TCO structures were analyzed and reported by various researchers. Yun et al. reported that the combination of $\mathrm{ITO} / \mathrm{Ni} / \mathrm{AZO}$ enhanced the electrical conductivity, mobility, and the carrier concentration [11]. Other combinational structures having different metal layers such as ITO/Au/ITO [12], TCO/Ag/ TCO [13-15], AZO/Mo/AZO [16], and AZO/Au/AZO [17] were also reported. Each combination has its own individuality in enhancing overall performance of the device. Chua et al. reported that the multilayer structure of $\mathrm{ZnO} / \mathrm{Ag} / \mathrm{ZnO}$ having high transmittance of $90 \%$ at $580 \mathrm{~nm}$ and low sheet resistance of $3 \Omega /$ sq. was fabricated by the Magnetron Sputtering [18]. Sahu and Huang reported the multilayer electrodes of ITO/Ag/ITO achieving a low sheet resistance below $6 \Omega /$ sq. and an optical transparency above $90 \%$ with overall thickness value of $100 \mathrm{~nm}$ at room temperature conditions by sputtering [19]. These reports indicate that the enhancement of the $\mathrm{Ag}$ inserted heterojunction structures for PV devices. Besides, Ag film is chosen to improve the electrical properties of the electrodes further because Ag has the lowest resistivity of all metals for bulk materials under normal conditions [20-23].

In this work, we have presented the improved electrical and optical properties of ITO/Ag/AZO-trilayered photodetectors. A thin Ag layer is inserted between the top ITO and bottom AZO layers. The design was carried out by depositing the three layers, step by step. The optical and electrical properties of ITO/Ag/AZO sample were compared with $\mathrm{AZO}$ and $\mathrm{Ag} / \mathrm{AZO}$ layers within the overall conditional thickness of $100 \mathrm{~nm}$. Here, the interdependence between the electrical and optical properties of single-layer and multilayers has been investigated as a function of the film thickness and deposition time with the objective of developing highperformance transparent conductive electrodes by sputtering technique at room temperature. This study provides a new design for improved quality of TCO thin films in optoelectronic applications at a low cost.

\section{Experimental Procedure}

Prior to the growth of ITO/Ag/AZO, n-type silicon (Si) substrates were chemically cleaned by the ultrasonication method to avoid any contamination on the surface. AZO film was deposited on $\mathrm{n}$-Si substrate by a sputtering system (SNTEK, Korea) at an RF power of $300 \mathrm{~W}$. Then, a thin Ag layer was deposited over AZO layer by applying DC power of $50 \mathrm{~W}$ to the Ag (99.99\%) target. Following this, a DC power source of $300 \mathrm{~W}$ was applied to an ITO target to form a layer upon the Ag film. Sputtering process has been performed at room temperature in an Argon atmosphere that was adjusted during the layer (ITO/Ag/AZO) deposition with varying the sputtering time for each layer. Aluminum (Al) grid was introduced on the top ITO layer, and the $\mathrm{Al}$ back contact was formed with $\mathrm{Si}$ as Ohmic electrodes. Both the prepared samples were annealed at $300^{\circ} \mathrm{C}$ by rapid thermal annealing process for 10 minutes to increase the crystallinity of the sample device.

A field emission scanning electron microscope (FE-SEM, FEI Sirion) was used to observe the cross-sectional and top view images of the prepared samples. The crystal structures and preferential orientation of the TCO and Ag films were analyzed by X-ray diffraction (XRD, Rigaku, D/Max 2500) with $\mathrm{Cu} \mathrm{K}-\alpha$ radiation in $\theta-2 \theta$ scan mode. Optical transmittance studies were observed for the samples deposited on glass substrate with the UV spectrophotometer (Scinco, Neosys-2000). The electrical and optical properties were achieved using a probe station with the measuring instruments (Keithley 2440).

\section{Results and Discussion}

Three different samples of AZO $(100 \mathrm{~nm}) / \mathrm{n}-\mathrm{Si}, \mathrm{Ag}(5 \mathrm{~nm}) /$ AZO $(95 \mathrm{~nm}) / \mathrm{n}-\mathrm{Si}$, and ITO $(45 \mathrm{~nm}) / \mathrm{Ag}(5 \mathrm{~nm}) / \mathrm{AZO}$ $(50 \mathrm{~nm}) / \mathrm{n}$-Si upon $\mathrm{n}$-Si substrate are prepared which are schematically presented in Figure 1(a). The three devices were fabricated such that the optimum thickness was fixed for all the three devices, that is, overall $100 \mathrm{~nm}$ for each device. The thickness of each material measured at 10 points by FE-SEM. The thickness error range is shown in Figure 1(b) and Table 1.

The surface morphology of the three samples was examined using FE-SEM analysis. Figures 2(a), 2(b), 2(c), 2(d), 2(e), and 2(f) show the cross-sectional and top view images of samples AZO (S1), Ag/AZO (S2), and ITO/Ag/AZO (S3). In the cross-sectional images of the sample S1 (Figure 2(a)), the continuous growth of $100 \mathrm{~nm}$ thick AZO layer was observed whereas in the sample S2 (Figure 2(b)), a thin layer of $\mathrm{Ag}$ is formed. In the sample S3 (Figure 2(c)), the insertion of Ag layer embedded between the ITO and AZO layers was seen. The layer thickness and metal insertion play an important role to determine the decrease in the sheet resistance. $\mathrm{Wu}$ and $\mathrm{Chu}$, Boscarino et al., and Kim et al. reported that a thin Ag layer revealed the lowering of sheet resistance $([13,16]$ and [24]). In the top view of the prepared samples as seen in Figures 2(d), 2(e), and 2(f), a uniform distribution of grains on the surface was observed. The grain size in the sample S3 (Figure 2(f)) was increased when compared to the S1 and S2 samples (Figures 2(d) and 2(e)).

The combination of metal and substrate is determined with indentation response. The relationship is given as follows:

$$
\begin{aligned}
\frac{1}{E_{r}}= & \frac{1-v_{i}^{2}}{E_{i}}+\frac{1-v_{f}^{2}}{E_{f}}\left(1-e^{-(\alpha(t-h)) / \sqrt{A}}\right) \\
& +\frac{1-v_{s}^{2}}{E_{s}}\left(e^{-(\alpha(t-h)) / \sqrt{A}}\right),
\end{aligned}
$$

where $E_{r}$ is elastic modulus, $E_{f}$ and $v_{f}$ are the modulus and Poisson's ratio of metal film, $E_{s}$ and $v_{s}$ are the modulus and Poisson's ratio of substrate, and $t$ and $h$ are the metal film thickness and depth. Metal film thickness is the important parameter to decide indentation size. Generally speaking, as 


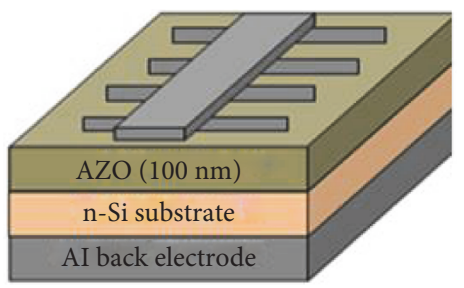

AI back electrode
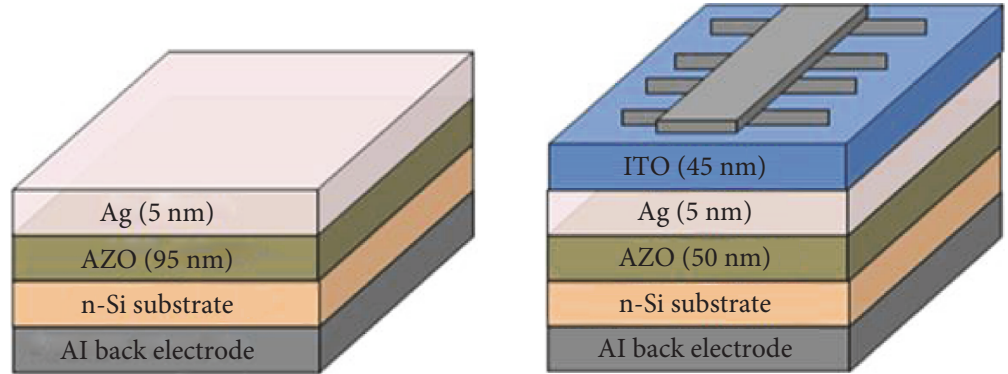

(a)

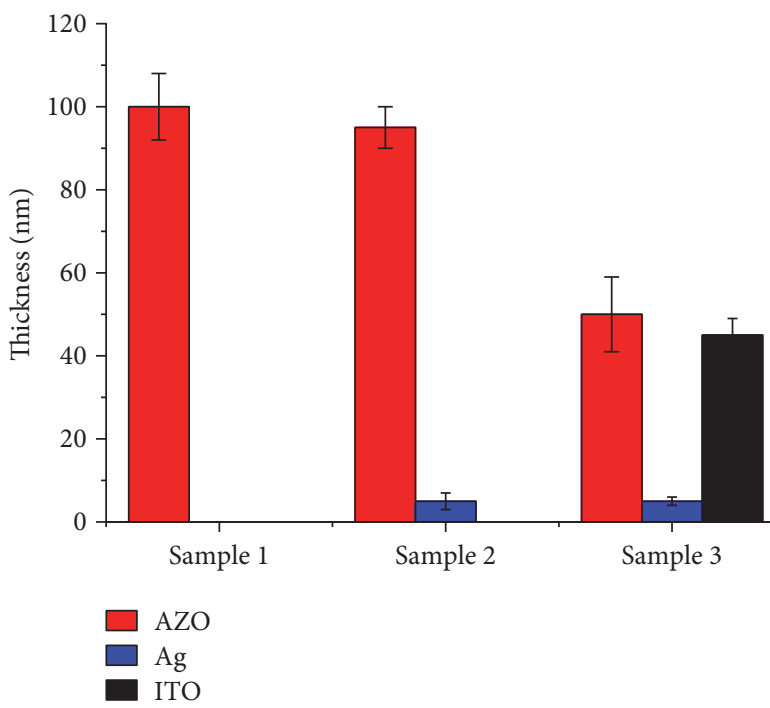

(b)

Figure 1: Schematic representation of the samples: (a) AZO/n-Si represented as S1, Ag/AZO/n-Si represented as S2, and ITO/Ag/AZO/n-Si represented as $\mathrm{S} 3$.

TABLE 1: The thickness and error range of AZO, Ag, and ITO.

\begin{tabular}{lccc}
\hline & $\begin{array}{c}\text { AZO thickness } \\
(\mathrm{nm})\end{array}$ & $\begin{array}{c}\text { Ag thickness } \\
(\mathrm{nm})\end{array}$ & $\begin{array}{c}\text { ITO thickness } \\
(\mathrm{nm})\end{array}$ \\
\hline Sample 1 & $100 \pm 8$ & - & - \\
Sample 2 & $95 \pm 5$ & $5 \pm 1$ & - \\
Sample 3 & $50 \pm 9$ & $5 \pm 2$ & $45 \pm 4$ \\
\hline
\end{tabular}

the metal thickness increases, strain gradient of metal increases. By contrast, yield strength decreases. True stresstrue strain relationship is given as follows:

$$
\sigma=\left(\begin{array}{cc}
E_{\varepsilon} & \varepsilon<\frac{\sigma_{y}}{E} \\
\sigma_{y}\left(\frac{\varepsilon}{\sigma_{y} / E}\right)^{N} & \varepsilon>\frac{\sigma_{y}}{E}
\end{array}\right)
$$

where $\sigma_{y}$ is the yield stress and $E$ is the modulus. The grain size is dependent to the yield strength, and this relationship is well known by Hall-Petch relationship. This is given as follows:

$$
\sigma_{y}=\sigma_{0}+\frac{k}{\sqrt{d}},
$$

where $d$ is the grain size and $\sigma_{0}$ is the intrinsic yield strength [25-27]. The Ag layer is applied to the transparent electrode by seed layer in this research. We can control the grain size by changing the Ag layer thickness, and which is seed layer to improve the properties of the transparent electrode by depositing ITO on Ag seed layer.

The grain size was measured by XRD analysis, and it is shown in Figure 3. By specifying the size of individual grains as symbol $D$, the size distribution of many crystallites can be approximated by the distribution function $g$ $(D)$. For a given sample, the particle size distribution function $g(D)$ is generally quite different from the thermal length distribution function $p(L)$. Converting $p(L)$ to $g(D)$ requires knowledge of the shape of each crystal since the distribution of the column lengths of a given grain is related to the geometric boundaries of the grain. 


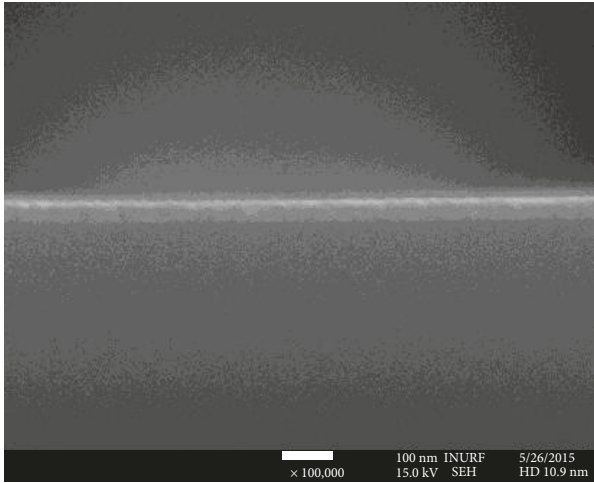

(a)

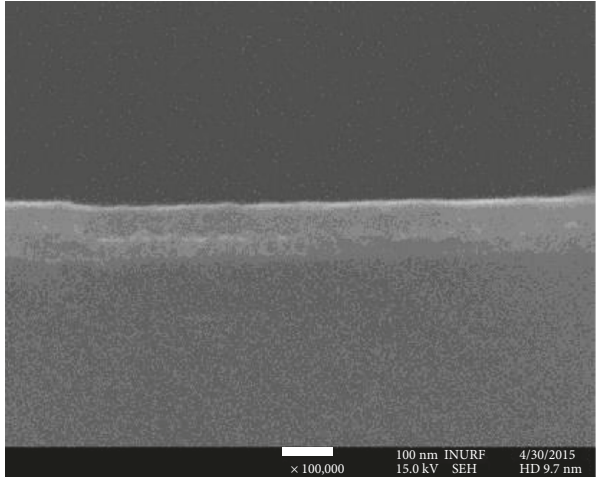

(c)

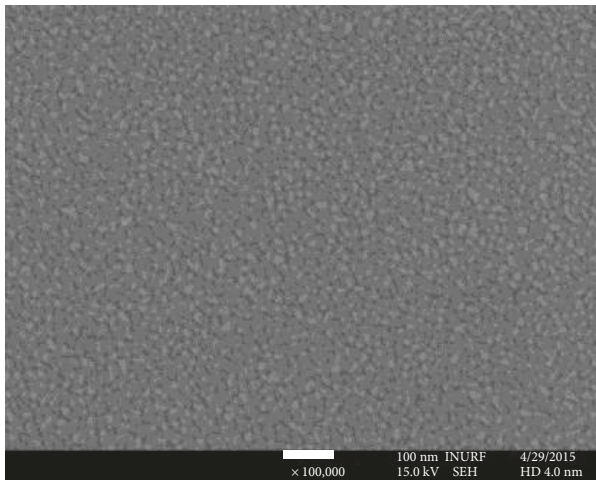

(e)

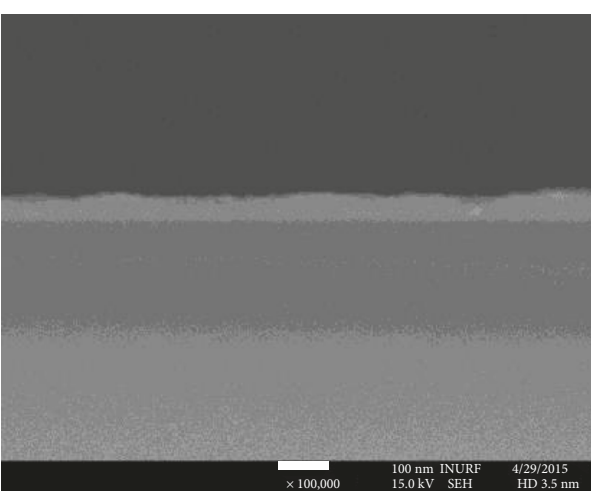

(b)

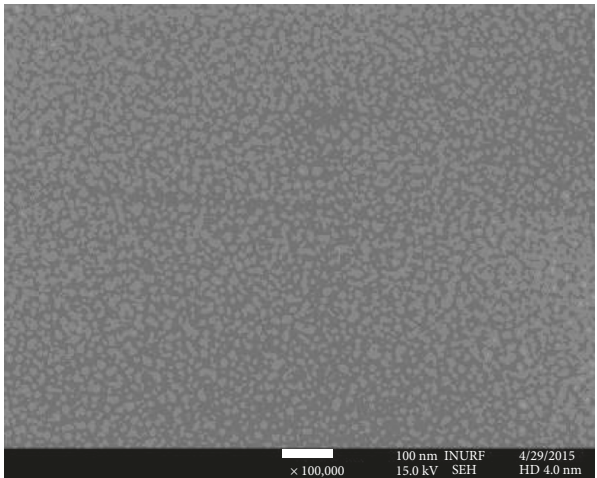

(d)

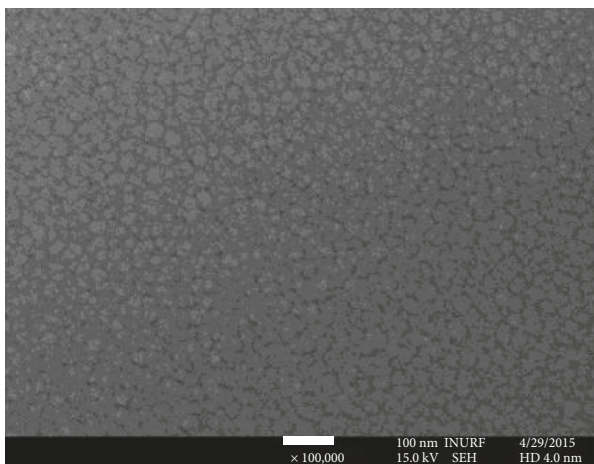

(f)

FIgUre 2: Cross-sectional FE-SEM images of (a) AZO, (b) Ag/AZO, and (c) ITO/Ag/AZO. Top view FE-SEM images of (a) AZO, (b) Ag/AZO, and (c) ITO/Ag/AZO.

The general relation between the two distributions is given as follows:

$$
\begin{array}{r}
p(L)=N \int_{0}^{\infty} f(L, D) g(D) d D, \\
f_{\text {sphere }}(L, D)=\left\{\begin{array}{cl}
\frac{\pi}{2} L & \text { if } L<<D, \\
0 & \text { if } L>D,
\end{array}\right.
\end{array}
$$

where $N$ is the normalization factor and $f\left(L_{0}, D_{0}\right) d L$ is the number of columns in the single crystal of size $D_{0}$, perpendicular to the reflecting surface, with a length between $L_{0}$ and $L_{0}$ $+d L$. If $D_{0}$ is too small to accommodate the columns which assumed length is $L_{0}, f\left(L_{0}, D_{0}\right) d L=0$. As you can see below the length distribution, $p(L)$ is contained in Bragg peak profile's Fourier coefficient to be measured in the diffraction experiment. The Bragg peak profile is expressed as a Fourier series. It is given as follows:

$f(s) \propto \sum_{L=-\infty}^{\infty}\left\{A(L) \cos \left[2 \pi\left(s-s_{0}\right) L\right]+B(L) \sin \left[2 \pi\left(s-s_{0}\right) L\right]\right\}$,

where $L$ is the conjugate variable to $\left(s-s_{0}\right)$ and $A(L)$ and $B(L)$ are the cosine and sine Fourier coefficients. $s$ is a function of the diffraction angle $\theta$ and the X-ray wavelength $\lambda . s$ is expressed as $(2 \sin \theta) / \lambda$. 


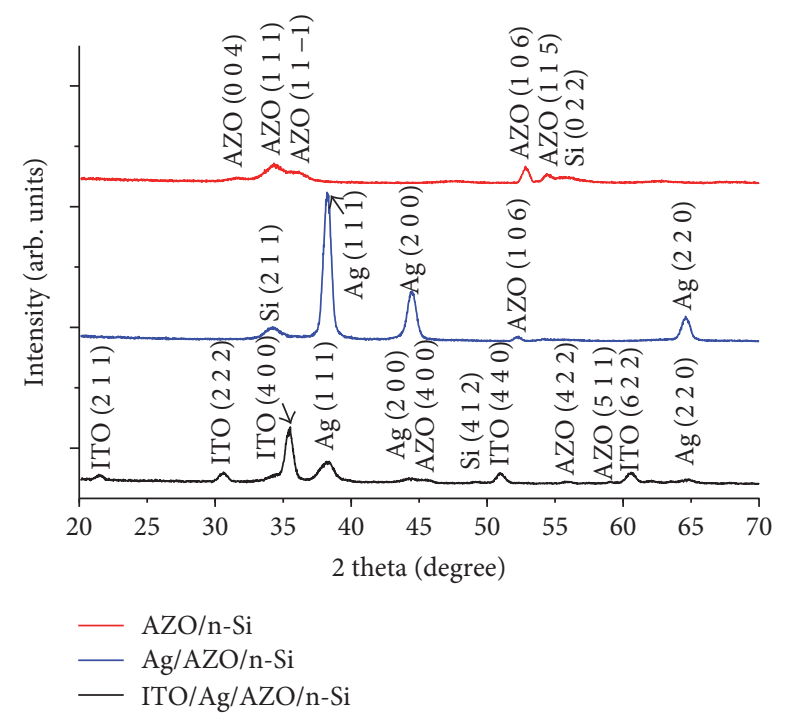

Figure 3: XRD patterns of the single AZO film, Ag/AZO film, and ITO/Ag/AZO film deposited on n-Si.

The average grain size $(D)_{\text {area }}$ and the volume-weighted average grain size $(D)_{\text {vol }}$ for a normal distribution are given as follows:

$$
\begin{aligned}
& (D)_{\text {area }}=D_{0} \exp \left(\frac{5}{2}(\ln \sigma)^{2}\right), \\
& (D)_{\mathrm{vol}}=D_{0} \exp \left(\frac{7}{2}(\ln \sigma)^{2}\right) .
\end{aligned}
$$

The width of $g(D)$ is gained from the ratio of $(D)_{\mathrm{vol}}$ to $(D)_{\text {area }}[28-30]$.

The structural properties of the $\mathrm{AZO}, \mathrm{Ag} / \mathrm{AZO}$, and ITO/ $\mathrm{Ag} / \mathrm{AZO}$ samples were analyzed using X-ray diffraction (XRD, Rigaku, D/Max 2500) with $\mathrm{Cu}$ K- $\alpha$ radiation in $\theta-2 \theta$ scan mode. Figure 3 shows the XRD patterns of the three samples. The single AZO layer of $100 \mathrm{~nm}$ thickness shows $\left(\begin{array}{lll}1 & 1 & 1\end{array}\right)$ and $\left(\begin{array}{lll}1 & 0 & 6\end{array}\right)$ peaks at around $36.71^{\circ}$ and $54.72^{\circ}$, respectively. From the Scherrer's equation, the grain size was calculated and obtained as $5.22 \mathrm{~nm}$ and $2.974 \mathrm{~nm}$ for the $\left(\begin{array}{lll}1 & 1 & 1\end{array}\right)$ and $\left(\begin{array}{lll}1 & 0 & 6\end{array}\right)$ peaks, respectively. In the Ag/AZO sample, $\mathrm{Ag}\left(\begin{array}{lll}1 & 1 & 1\end{array}\right)$ peak is observed at $38.23^{\circ}$ with a grain size of $0.125 \mathrm{~nm}$ with a cubic structure [31]. And in the third sample device, that is, ITO/Ag/AZO, peaks were observed for ITO (2 22 ) at $30.60^{\circ}$, ITO (4 00$)$ at $35.40^{\circ}$, ITO (4 40 ) at $50.89^{\circ}$, and ITO $\left(\begin{array}{ll}6 & 2\end{array}\right)$ at $60.52^{\circ}$. The average grain size was calculated by using XRD peaks to find $0.1128 \mathrm{~nm}$, $0.176 \mathrm{~nm}, 0.1167 \mathrm{~nm}$, and $0.1058 \mathrm{~nm}$ for ITO (2 22$)$, ITO (4 0 0), ITO (4 4 0), and ITO (6 22 2) planes, respectively. The Ag (llll) at $38.22^{\circ}$ and AZO (5 111$)$ at $60.52^{\circ}$ peaks were also noted along with the ITO peaks $[20,31]$.

The optical quality of the AZO, Ag/AZO, and ITO/Ag/ AZO thin films based on its transparency was examined with the aid of UV-visible spectrophotometer, as shown in Figure 4. The transmittance spectra of $\mathrm{AZO}, \mathrm{Ag} / \mathrm{AZO}$, and ITO/Ag/AZO with a reference glass sample are depicted in Figure 4 as a function of wavelength. The optical transmittance of the glass substrate is about $85 \%$ over the visible range of wavelength. The average value of transmittance for AZO, $\mathrm{Ag} / \mathrm{AZO}$, and ITO/Ag/AZO samples are $79.21 \%, 31.37 \%$, and $40.96 \%$, respectively. Accordingly, the Ag/AZO bilayer shows a lower transmittance characteristic as compared to the AZO single layer and ITO/Ag/AZO trilayer. This is mainly due to the Ag layer as Ag is known for its light scattering phenomena especially in the wavelength range beyond $450 \mathrm{~nm}$. These transmittance characteristics can be compared with the results reported by Sahu and Huang [19], Boscarino et al. [13], Jung et al. [14], and Ren et al. [15] for the trilayered photodetectors. The sheet resistance measured using the probe method for the AZO, $\mathrm{Ag} / \mathrm{AZO}$, and ITO/Ag/ AZO samples was $23.65 \Omega /$ sq., $13.962 \Omega /$ sq., and $1.106 \Omega /$ sq., respectively. The increased carrier concentration is the reason for highly reduced sheet resistance observed in Aginserted sample. The figure of merit $\left(\Phi_{\mathrm{TC}}\right)$ is an important factor which evaluates the overall performance of TCO materials and is given [32] as follows:

$$
\Phi_{\mathrm{TC}}=\frac{T^{10}}{R_{\mathrm{s}}},
$$

where $T$ is the transmittance and $R_{\mathrm{s}}$ is the sheet resistance. The calculated figure of merit (FOM) for $\mathrm{AZO}, \mathrm{Ag} / \mathrm{AZO}$, and ITO/Ag/AZO is $1.6640 \times 10^{-3} \Omega^{-1}, 6.6099 \times 10^{-7} \Omega^{-1}$, and $1.2018 \times 10^{-4} \Omega^{-1}$, respectively, at a wavelength of $480 \mathrm{~nm}$. The higher FOM indicates that the ITO/Ag/AZOlayered device offers enhanced optoelectrical properties than $\mathrm{AZO}-$ and Ag/AZO-layered devices. ITO was deposited on $\mathrm{Ag}$ seed layer for reducing reflectance, because ITO has properties that are high transmittance and low reflectance. Although Ag layer cause high reflectance, it can reduce sheet resistance and by depositing ITO on Ag layer, reflectance can be decreased, because the light to reflect at $\mathrm{Ag}$ layer is transmitted across the device again due to light scattering phenomenon. We can confirm that the transmittance and reflectance are improved at $\mathrm{ITO} / \mathrm{Ag} / \mathrm{AZO} / \mathrm{n}$-Si device 


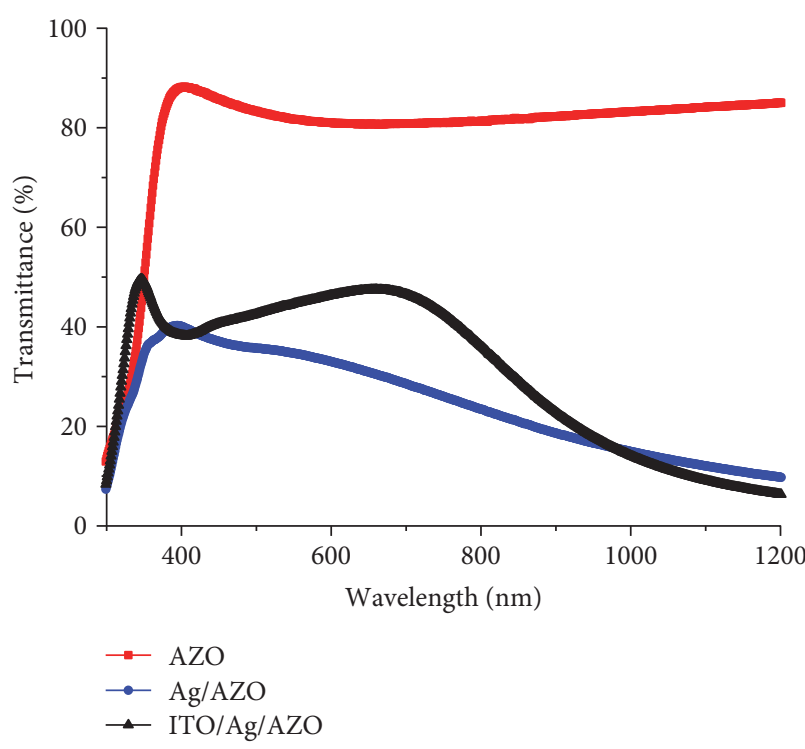

(a)

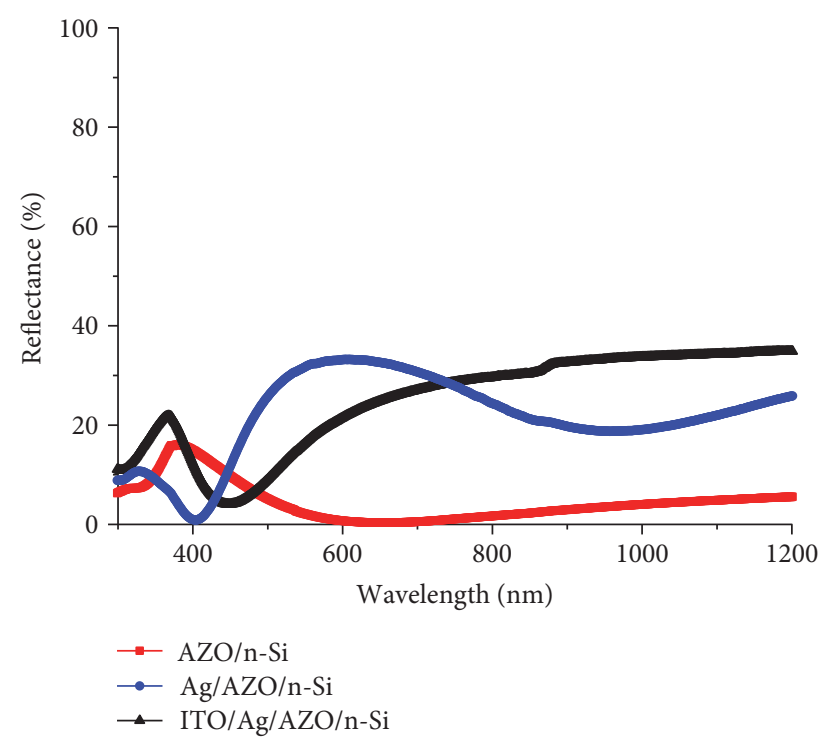

(b)

Figure 4: Optical property spectra of AZO, Ag/AZO, and ITO/Ag/AZO devices; (a) transmittance and (b) reflectance.
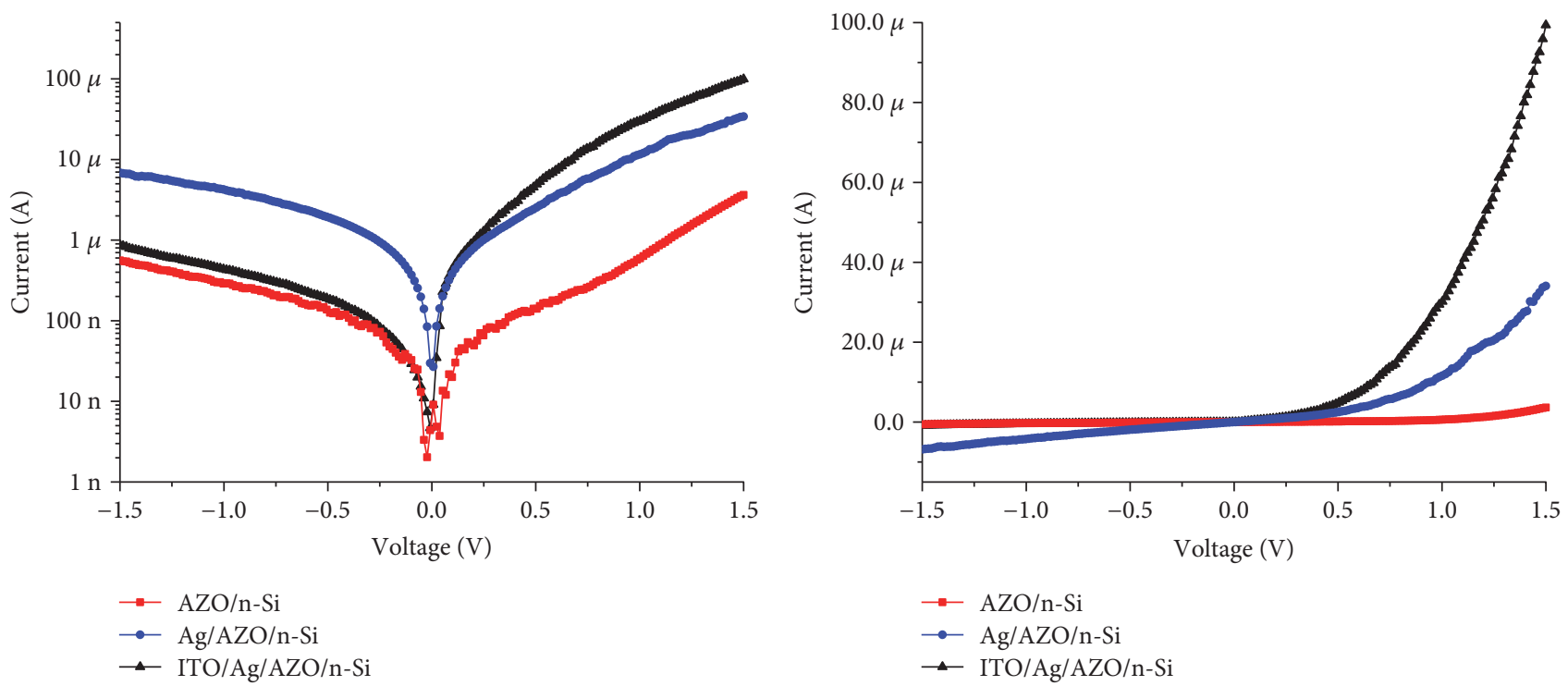

FIgURE 5: Dark I-V profiles of S1, S2, and S3 devices.

TABLE 2: The characterized electrical and optical parameters of AZO, Ag/AZO, and ITO/Ag/AZO devices.

\begin{tabular}{|c|c|c|c|c|c|}
\hline Sample device & Thickness (nm) & Transmittance (\%) & Sheet resistance ( $\Omega$ /sq.) & Figure of merit & Rectifying ratio \\
\hline $\mathrm{AZO}$ & $100 \mathrm{~nm}$ & 72.36 & 23.65 & $1.6640 \times 10^{-3} \Omega^{-1}$ & 6.49 \\
\hline $\mathrm{Ag} / \mathrm{AZO}$ & $5 \mathrm{~nm} / 95 \mathrm{~nm}$ & 31.37 & 13.962 & $6.6099 \times 10^{-7} \Omega^{-1}$ & 4.97 \\
\hline ITO/Ag/AZO & $45 \mathrm{~nm} / 5 \mathrm{~nm} / 50 \mathrm{~nm}$ & 40.96 & 1.106 & $1.2018 \times 10^{-4} \Omega^{-1}$ & 113.38 \\
\hline
\end{tabular}

compared to them at $\mathrm{Ag} / \mathrm{AZO} / \mathrm{n}-\mathrm{Si}$ device. The reflectance profile of $\mathrm{AZO}, \mathrm{Ag} / \mathrm{AZO}$, and ITO/Ag/AZO samples was shown in Figure 4. The enhanced absorption and reduced reflection are the two most important factors for highly efficient photo devices [33]. The average reflectance of prepared samples of $\mathrm{AZO}, \mathrm{Ag} / \mathrm{AZO}$, and $\mathrm{ITO} / \mathrm{Ag} / \mathrm{AZO}$ was $4.26 \%, 25.11 \%$, and $15.69 \%$ (400 $\mathrm{nm}-700 \mathrm{~nm}$ ), respectively.
The fabricated AZO/n-Si, Ag/AZO/n-Si, and ITO/Ag/ $\mathrm{AZO} / \mathrm{n}$-Si heterojunction photodetectors were characterized to examine the diode performance under the dark as shown in Figure 5. The samples were deposited with the aluminum (Al) grid on the top surface. Al was also sputtered on the Si substrate as a back electrode. For a photovoltaic device performance, it is important to form a 

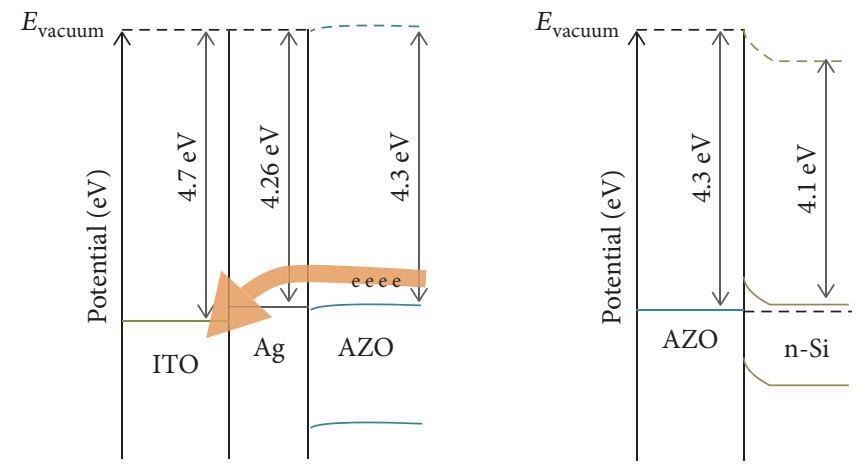

FIgURE 6: Band diagram of ITO/Ag/AZO/n-Si device.
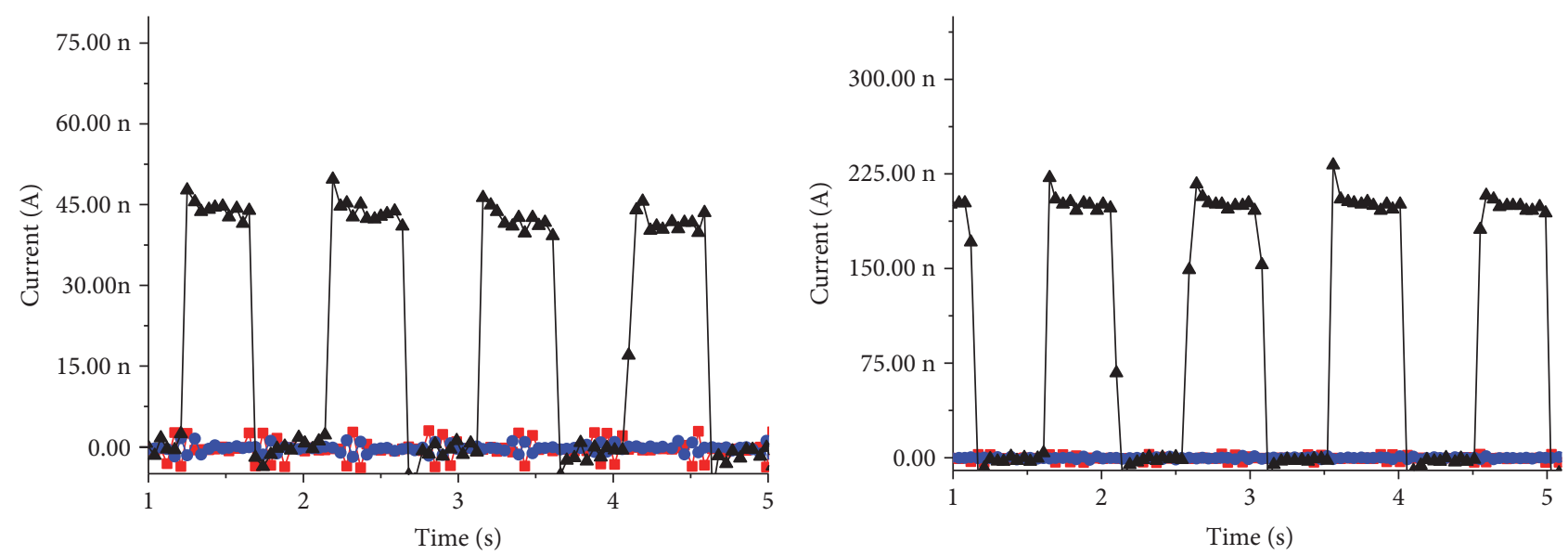

Wavelength: $380 \mathrm{~nm}$

$$
\begin{aligned}
& \text { Wavelength: } 600 \mathrm{~nm} \\
& \rightarrow-\mathrm{AZO} \\
& \rightarrow \mathrm{Ag} / \mathrm{AZO} \\
& -\mathrm{ITO} / \mathrm{Ag} / \mathrm{AZO}
\end{aligned}
$$

(a)
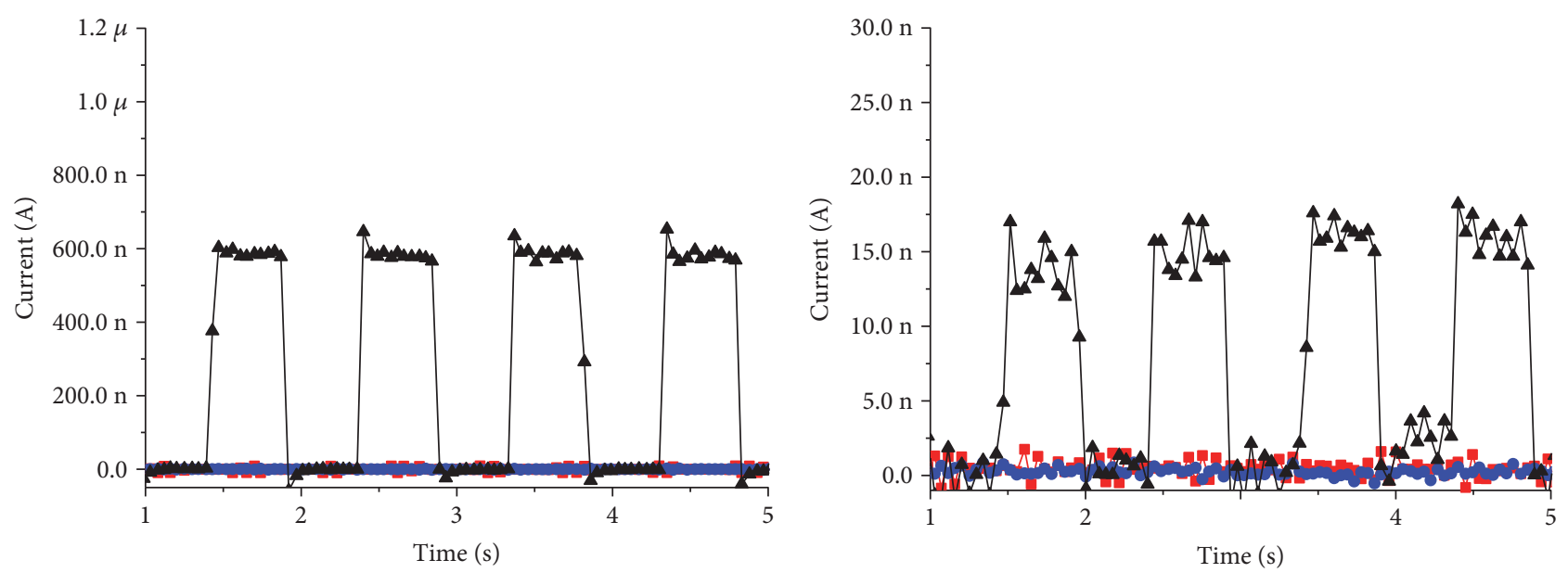

Wavelength: $900 \mathrm{~nm}$

$$
\begin{aligned}
& \because \mathrm{AZO} \\
& \because \mathrm{Ag} / \mathrm{AZO} \\
& \because \mathrm{ITO} / \mathrm{Ag} / \mathrm{AZO}
\end{aligned}
$$

Wavelength: $1100 \mathrm{~nm}$

$$
\begin{aligned}
& \because \mathrm{AZO} \\
& \because \mathrm{Ag} / \mathrm{AZO} \\
& -\mathrm{ITO} / \mathrm{Ag} / \mathrm{AZO}
\end{aligned}
$$

(c)

(d)

FIgure 7: Photoresponses of AZO, Ag/AZO, and ITO/Ag/AZO devices at a wavelength of (a) $380 \mathrm{~nm}$, (b) $600 \mathrm{~nm}$, (c) $900 \mathrm{~nm}$, and (d) $1100 \mathrm{~nm}$. 
quality junction; otherwise, the photovoltaic effect will be faded away by recombination in advance of collecting the photo-generated carriers [7]. The heterojunction AZO/n-Si gave a clear rectifying current flow with the rectifying ratio of 6.49 which was higher than $\mathrm{Ag} / \mathrm{AZO} / \mathrm{n}-\mathrm{Si}$ with a rectifying ratio of 4.97 . But in the third sample, ITO/Ag/AZO/n-Si showed a high rectifying ratio of about 113.38 indicating an improvement in the quality of the junction device. The electrical and optical studies are described in Table 2.

Each material work function of ITO, AZO, and Ag is about $4.7 \mathrm{eV}, 4.26 \mathrm{eV}$, and $4.3 \mathrm{eV}$. The band diagram of ITO/Ag/AZO/n-Si is shown in Figure 6. ITO/Ag/AZO transparent electrode makes the electron accelerate and that makes result that the current increase at forward bias. Also, it reduces the saturation current by inhibiting the recombination of electron and hole $[34,35]$. As a result, Schottky curve is shown clearly at dark $\mathrm{I}-\mathrm{V}$ characteristics and rectifying ratio is improved.

The photoresponse studies of the AZO, Ag/AZO, and ITO/ $\mathrm{Ag} / \mathrm{AZO}$ devices are observed as shown in Figures 7(a), 7(b), $7(\mathrm{c})$, and $7(\mathrm{~d})$ for different wavelengths such as $380 \mathrm{~nm}$, $600 \mathrm{~nm}, 900 \mathrm{~nm}$, and $1100 \mathrm{~nm}$, respectively. At the wavelengths $380 \mathrm{~nm}, 600 \mathrm{~nm}$, and $900 \mathrm{~nm}$, the photoresponse of ITO/Ag/AZO was prominent when compared to AZO and $\mathrm{Ag} / \mathrm{AZO}$ devices, respectively. At wavelengths of $380 \mathrm{~nm}$ (Figure 7(a)), $600 \mathrm{~nm}$ (Figure 7(b)), and $900 \mathrm{~nm}$ (Figure 7(c)), the photoresponses of ITO/Ag/AZO were prominent than those of the AZO and Ag/AZO layers. But at a longer wavelength of $1100 \mathrm{~nm}$ (Figure $7(\mathrm{~d})$ ), the photoresponsive behavior of ITO/Ag/AZO-, Ag/AZO-, and AZO-layered devices was comparatively very low. In particular, the photoresponse of ITO/Ag/AZO was considerably lower than that of the $\mathrm{Ag} / \mathrm{AZO}$ and $\mathrm{AZO}$ devices at high wavelength region. Since, it is a characteristic feature of ITO layer that excites free carriers without creating electron hole pairs at longer wavelength of $1100 \mathrm{~nm}$, resulting in poor photoresponse [36]. But at wavelengths of $350 \mathrm{~nm}$ and $600 \mathrm{~nm}$, the photoresponse of ITO/Ag/AZO is much better than that of the AZO- and Ag/ AZO-layered devices. These two wavelengths are essential for most of the photoelectric applications [32]. It is noteworthy to mention that the ITO/Ag/AZO device exhibited the high photoresponse at the wavelength of $900 \mathrm{~nm}$ which is also essential for Si-based solar cells and photodetectors [36, 37]. When AZO, Ag/AZO, ITO/Ag/AZO were used as transparent electrodes, the ITO/Ag/AZO transparent electrode reduced the sheet resistance and minimized the reduction of the transmittance. As a result, it can be confirmed that the performance index (FOM) of the transparent electrode is improved. This means that the light penetrates through the transparent electrode and is sufficiently absorbed at the junction of the device and the electron transfer becomes easy due to the low sheet resistance. As a result, the photocurrent increases and it shows a remarkable improvement in the photoresponse.

\section{Conclusion}

In summary, the optical and electrical properties of Agembedded TCO layers were systematically analyzed with multilayered devices within the optimized thickness range of $100 \mathrm{~nm}$, that is, AZO $(100 \mathrm{~nm}) / \mathrm{n}-\mathrm{Si}, \mathrm{Ag}(5 \mathrm{~nm}) / \mathrm{AZO}$ $(95 \mathrm{~nm}) / \mathrm{n}-\mathrm{Si}$, and ITO $(45 \mathrm{~nm}) / \mathrm{Ag}(5 \mathrm{~nm}) / \mathrm{AZO}(50 \mathrm{~nm}) / \mathrm{n}-$ Si. The optical transmittance and electrical conductivity are the two important features for any transparent conductive electrode designs. The ITO/Ag/AZO sample revealed the better optical and electrical properties than AZO and Ag/AZO samples. When compared with $\mathrm{AZO}$ and $\mathrm{Ag} / \mathrm{AZO}$ layers deposited upon $\mathrm{n}-\mathrm{Si}$ substrate at room temperature, the Aginserted ITO/Ag/AZO-trilayered sample exhibited better results in both electrical and optical studies. Among all the three samples, Ag-inserted ITO/Ag/AZO sample showed an improvement in optoelectronic properties especially in the photoresponse studies. The ITO/Ag/AZO layer had an enhanced rectification ratio with a value of 113.38 , and its sheet resistance value was reduced to $1.106 \Omega /$ sq. Thus, the layers $\mathrm{AZO}$ and $\mathrm{Ag} / \mathrm{AZO}$, respectively, deposited upon $\mathrm{n}-\mathrm{Si}$ substrate were further improved satisfactorily for the electrical and optical properties by inserting Ag layer between the ITO and AZO layers. This result provides the advantageous features of ITO/Ag/AZO device for better performance in the photoelectric industry.

\section{Conflicts of Interest}

The authors declare that there is no conflict of interests regarding the publication of this paper.

\section{Authors' Contributions}

Gyeong-Nam Lee and Ponnamma Machaiah M. equally contributed to this work.

\section{Acknowledgments}

This research was supported by Basic Science Research Program through the National Research Foundation of Korea (NRF) by the Ministry of Education (NRF-2015R1 D1A1A01059165) and the Korea Institute of Industrial Technology as Convergence research (KITECH EO-17-0018).

\section{References}

[1] S.-H. Hong, J.-H. Yun, H.-H. Park, and J. Kim, "Nanodomepatterned transparent conductor for highly responsive photoelectric device," Applied Physics Letters, vol. 103, article 153504, 2013.

[2] J. Muller, B. Rech, J. Springer, and M. Vanecek, "TCO and light trapping in silicon thin film solar cells," Solar Energy, vol. 77, pp. 917-930, 2004.

[3] M. Melvin David Kumar, J.-H. Yun, and J. Kim, "Metal/ semiconductor and transparent conductor/semiconductor heterojunctions in high efficient photoelectric devices: progress and features," International Journal of Photoenergy, vol. 2014, Article ID 160379, 14 pages, 2014.

[4] http://www.energysavers.gov/yourhome/windowsdoorssky light $/$ index.cfm/mytopic $=13430$.

[5] T. Koida, H. Fujiwara, and M. Kondo, "High-mobility hydrogen-doped $\operatorname{In}_{2} \mathrm{O}_{3}$ transparent conductive oxide for a-Si: $\mathrm{H} / \mathrm{c}-\mathrm{Si}$ heterojunction solar cells," Solar Energy Materials \& Solar Cells, vol. 93, pp. 851-854, 2009. 
[6] J. H. Yun, J. Kim, Y. C. Park, S. J. Moon, and W. A. Anderson, "Double transparent conducting layers for Si photovoltaics," Thin Solid Films, vol. 547, pp. 17-21, 2013.

[7] B. H. Kong, M. K. Choi, H. K. Cho, J. H. Kim, S. Baek, and J. H. Lee, "Conformal coating of conductive $\mathrm{ZnO}$ : Al films as transparent electrodes on high aspect ratio $\mathrm{Si}$ microrods," Electrochemical Solid State, vol. 13, p. k12, 2010.

[8] S. W. Jee, S. J. Park, J. Kim et al., "Efficient three-dimensional nanostructured photoelectric device by $\mathrm{Al}-\mathrm{ZnO}$ coating on lithography-free patterned Si nanopillars," Applied Physics Letters, vol. 99, article 053118, 2011.

[9] J. H. Yun and J. Kim, "Double transparent conducting oxide films for photoelectric devices," Materials Letters, vol. 70, pp. 4-6, 2012.

[10] M. D. Kumar, Y. C. Park, and J. Kim, "Impact of thin metal layer on the optical and electrical properties of indiumdoped-tin oxide and aluminum-doped-zinc oxide layers," Superlattices and Microstructures, vol. 82, pp. 499-506, 2015.

[11] J.-H. Yun, N. Duraisamy, M. Melvin David Kumar, and J. Kim, "Optical and electrical properties of AZO/Ni/ITO transparent conductor," Materials Letters, vol. 143, pp. 215-218, 2015.

[12] Y. S. Kim, J. H. Park, D. H. Choi et al., "ITO/Au/ITO multilayer thin films for transparent conducting electrode applications," Applied Surface Science, vol. 254, pp. 15241527, 2007.

[13] S. Boscarino, I. Crupi, S. Mirabella, F. Simone, and A. Terrasi, "TCO/Ag/TCO transparent electrodes for solar cells application," Physica A, vol. 116, pp. 1287-1291, 2014.

[14] Y. S. Jung, Y. S. Park, K. H. Kim, and W. J. Lee, "Properties of $\mathrm{AZO} / \mathrm{Ag} / \mathrm{AZO}$ multilayer thin film deposited on polyethersulfone substrate," Transactions on Electrical and Electronic Materials, vol. 14, pp. 9-11, 2013.

[15] N. F. Ren, L. J. Huang, M. Zhoub, and B. J. Li, "Introduction of $\mathrm{Ag}$ nanoparticles and AZO layer to prepare AZO/Ag/FTO trilayer films with high overall photoelectric properties," Ceramics International, vol. 40, pp. 8693-8699, 2014.

[16] H. W. Wu and C. H. Chu, "Structural and optoelectronic properties of $\mathrm{AZO} / \mathrm{Mo} / \mathrm{AZO}$ thin films prepared by rf magnetron sputtering," Materials Letters, vol. 105, p. 65, 2013.

[17] Y. S. Lin and W. C. Tseng, "Effect of Al nanoparticles on the microstructure, electrical, and optical properties of $\mathrm{AZO} / \mathrm{Al} /$ AZO trilayer thin film," Journal of Electronic Materials, vol. 41, pp. 437-441, 2012.

[18] C. H. Chua, H. W. Wub, and J. L. Huang, "AZO/Au/AZO trilayer thin films for the very low resistivity transparent electrode applications," Materials Science and Engineering: B, vol. 186, pp. 117-121, 2014.

[19] D. R. Sahu and J.-L. Huang, "Design of $\mathrm{ZnO} / \mathrm{Ag} / \mathrm{ZnO}$ multilayer transparent conductive films," Materials Science and Engineering: B, vol. 130, pp. 295-299, 2006.

[20] C. Guillen and J. Herrero, “Transparent conductive ITO/Ag/ ITO multilayer electrodes deposited by sputtering at room temperature," Optics Communications, vol. 282, pp. 574-578, 2009.

[21] A. Kloppel, W. Kriegseis, B. Meyer et al., "Dependence of the electrical and optical behaviour of ITO-silver-ITO multilayers on the silver properties," Thin Solid Films, vol. 365, pp. 139$146,2000$.

[22] D. Griffiths, Introduction to Electrodynamics, p. 286, third edition, 1999, 1981.
[23] R. A. Serway, Principles of Physics, London: Saunders College Pub, Fort Worth, TX, USA, 2nd edition, 1998.

[24] J. Kim, M. M. D. Kumar, and S. M. Baek, "The influence of Ni layer and thickness of AZO layers on the optoelectronic properties of $\mathrm{AZO} / \mathrm{Ni} / \mathrm{AZO}$ tri-layer deposited at room temperature," Materials Letters, vol. 137, pp. 132-135, 2014.

[25] N. Lu, Z. Suo, and J. J. Vlassak, "The effect of film thickness on the failure strain of polymer-supported metal films," Acta Materialia, vol. 58, pp. 1679-1687, 2010.

[26] Y. Cao, S. Allameh, D. Nankivil, S. Sethiaraj, T. Otiti, and W. Soboyejo, "Nanoindentation measurements of the mechanical properties of polycrystalline $\mathrm{Au}$ and $\mathrm{Ag}$ thin films on silicon substrates: effects of grain size and film thickness," Materials Science and Engineering, vol. 427, pp. 232-240, 2006.

[27] J. Yu, X. Zhao, and Q. Zhao, "Effect of film thickness on the grain size and photocatalytic activity of the sol-gel derived nanometer $\mathrm{TiO}_{2}$ thin films," Journal of Materials Science Letters, pp. 1015-1017, 2000.

[28] C. E. Kril and R. Birringer, "Estimating grain-size distributions in nanocrystalline materials from X-ray diffraction profile analysis," Philosophical Magazine A, vol. 8610, 2017.

[29] A. K. Kulkarni, K. H. Schulz, T. S. Lim, and M. Khan, "Dependence of the sheet resistance of indium-tin-oxide thin films on grain size and grain orientation determined from X-ray diffraction techniques," Thin Solid Films, vol. 345, pp. 273-277, 1999.

[30] Y. Zhong, D. Ping, X. Song, and F. Yin, "Determination of grain size by XRD profile analysis and TEM counting in nano-structured $\mathrm{Cu}$," Journal of Alloys and Compounds, vol. 476, pp. 113-117, 2009.

[31] A. H. Ali and Z. Hassan, "Improved optoelectronics properties of ITO-based transparent conductive electrodes with the insertion of Ag/Ni under-layer," Applied Surface Science, vol. 315, pp. 387-391, 2014.

[32] J.-H. Yun, M. Melvin David Kumar, and J. Kim, “Transparent conductors with an ultrathin nickel layer for high-performance photoelectric device applications," Materials Science in Semiconductor Processing, vol. 31, pp. 334-339, 2015.

[33] J. Y. Lee and J. W. Yang, "Dependence of intermediated noble metals on the optical and electrical properties of ITO/metal/ ITO multilayers," Optics Communications, vol. 282, no. 12, pp. 2362-2366, 2009.

[34] K. Ritzau, M. Bivour, S. Schröer et al., "TCO work function related transport losses at the a-Si:H/TCO-contact in SHJ solar cells," Solar Energy Materials \& Solar Cells, vol. 131, pp. 9-13, 2014.

[35] S. Ma, J. Xue, Y. Zhou, and Z. Zhang, "Photochemical synthesis of $\mathrm{ZnO} / \mathrm{Ag}_{2} \mathrm{O}$ heterostructures with enhanced ultraviolet and visible photocatalytic activity," Journal of Materials Chemistry A, vol. 2, pp. 7272-7280, 2014.

[36] Z. Holman, L. Barraud, and J. Seif, "Current losses at the front of silicon heterojunction solar cells," Journal of Photovoltaics, vol. 2, p. 7, 2012.

[37] S. Calnan and A. N. Tiwari, "High mobility transparent conducting oxides for thin film solar cells," Thin Solid Films, vol. 518, pp. 1839-1849, 2010. 

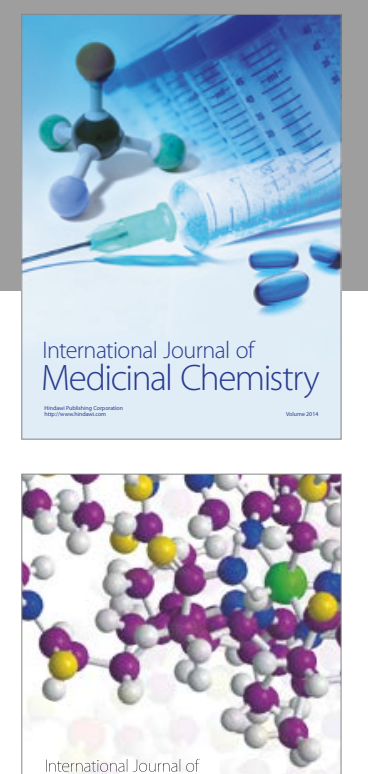

Carbohydrate Chemistry

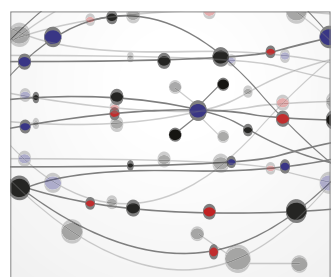

The Scientific World Journal
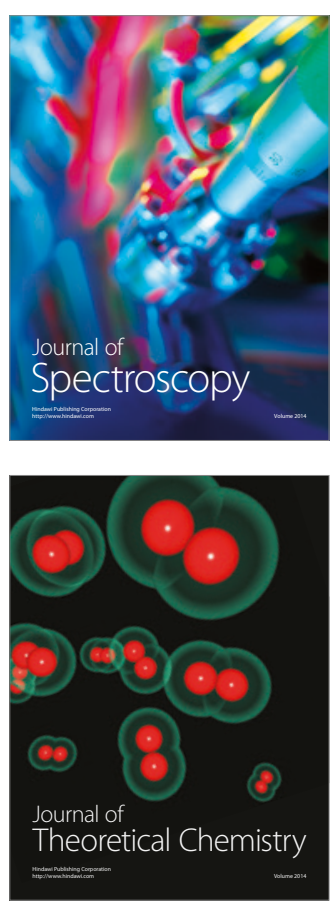
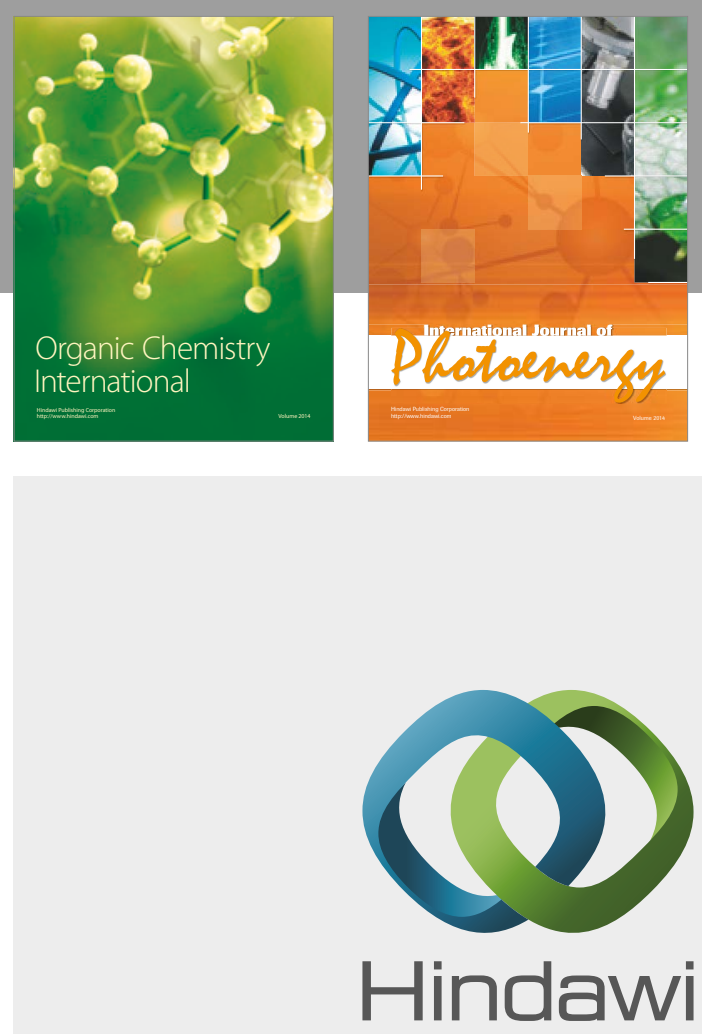

Submit your manuscripts at

https://www.hindawi.com

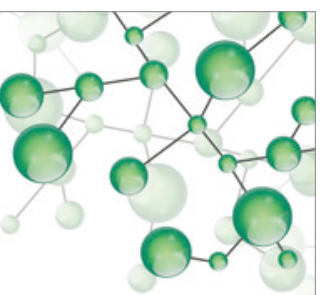

International Journal of

Inorganic Chemistry

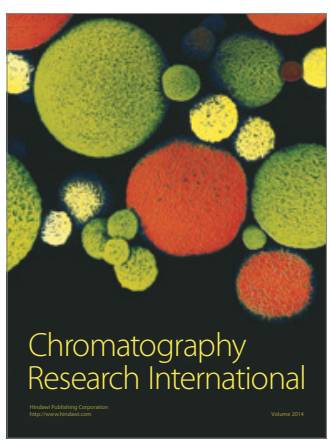

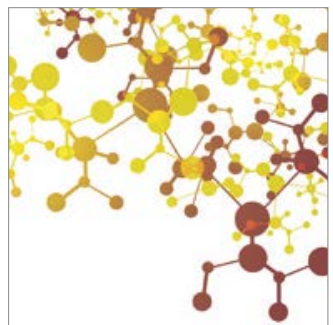

Applied Chemistry
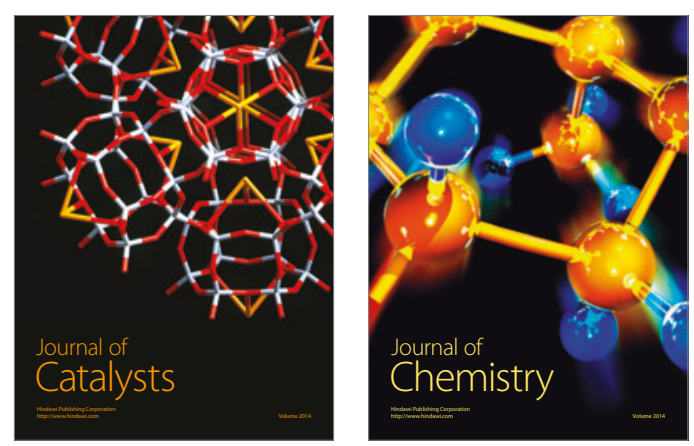
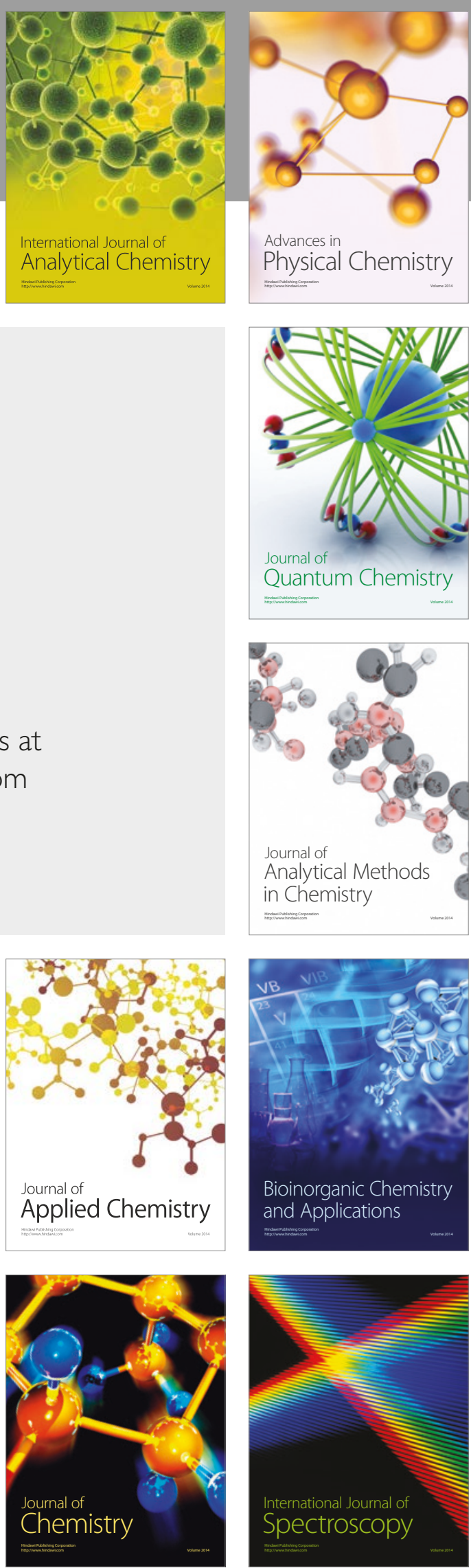